\title{
A Rare Case Report of Malignant Meningioma
}

\author{
Dr. Nihaal K Reddy ${ }^{1}$, Dr.Jithan Veigas ${ }^{2}$, Dr. Pradeep H Patil ${ }^{3}$ \\ ${ }^{1}$ (Department of Radiodiagnosis, KLE University, India) \\ ${ }^{2}$ (Department of Radiodiagnosis, KLE University, India) \\ ${ }^{3}$ (Department of Radiodiagnosis, KLE University, India)
}

\begin{abstract}
:
Aims \& Objectives: Atypical and malignant meningiomas are uncommon tumors with aggressive behavior and higher mortality, morbidity, and recurrence compared with benign tumors. We report a case report of malignant meningioma.

Materials \& Methods: A 53 year old male patient, presented with history large ulcerative swelling over the scalp for the past 5 years, with complaints of altered and aggressive behaviour and headache. Patient gives history of operation for benign meningioma 4 years back.Patient underwent MRI \& CT Brain plain and contrast.

Result: Patient underwent MRI \& CT Brain plain and contrast, showed a large heterogeneously enhancing extraxial mass lesion with irregular margins, significant extracalvarial spread and mushrooming into the brain parenchyma in the temporal \& parietal regions. The lesion showed intratumoral cysts within it.

On MRS significant choline, lipid and lactate peaks with near total dampening of $N A A, C r$, MI peaks.

The mean $A D C$ values were $(0.5-0.8 \times 10-3 \mathrm{~mm} 2 / \mathrm{s})$ was suggesting a high grade cellular lesion.

Conclusion: Anaplastic or malignant meningioma (MMen) corresponds histologically to WHO grade III.

Frankly malignant meningiomas are rare, representing only 1-3\% of all meningiomas. MMens have a striking male predominance.

The imaging triad of extracranial mass, osteolysis, and "mushrooming" intracranial tumor is present in most but not all cases of Malignant meningiomas.
\end{abstract}

Key-words: Malignant meningioma, mushrooming,extra-axial

\section{Introduction:}

Meningiomas are the most common intradural, extra-axial lesions of the CNS, accounting for $15 \%$ to $18 \%$ of intracranial neoplasms in adults.

Malignant meningiomas though are very rare and account for around $1 \%$ of all the meningiomas.

We report a large exophytic meningioma with malignant degeneration ( $1 \%$ incidence ) and intratumoral cysts ( $4-5 \%)$.

\section{Case Report:}

Patient underwent MRI \& CT Brain plain and contrast, showed a large heterogeneously enhancing extraxial mass lesion along the convexity in the left temporo-parietal region with irregular margins ,significant extracalvarial spread through the craniotomy site and mushrooming into the brain parenchyma in the temporal \& parietal regions. The lesion showed intratumoral cysts within it.

The lesion was predominantly isointense on T1,T2 and FLAIR images with interspersed heterogeneity. There was no evidence of calcification or flow voids within the lesion.

On MRS significant choline, lipid and lactate peaks with near total dampening of NAA, Cr, MI peaks.

The mean ADC values were $(0.5-0.8 \times 10-3 \mathrm{~mm} 2 / \mathrm{s})$ was suggesting a high grade cellular lesion.

On CT angiography, there was early filling of the venous channels.

Few dilated tortuous vessels were also seen along the convexity of the lesion in the extracalvarial portion.

Porencephalic cyst was seen in the left tempora-parietal regions due to old insult adjacent to the lesion. Biopsy of the lesion showed fibroblastic type of malignant meningioma.

FNAC of the cervical lymph node showed possibility of metastasis. 


\section{Figures}

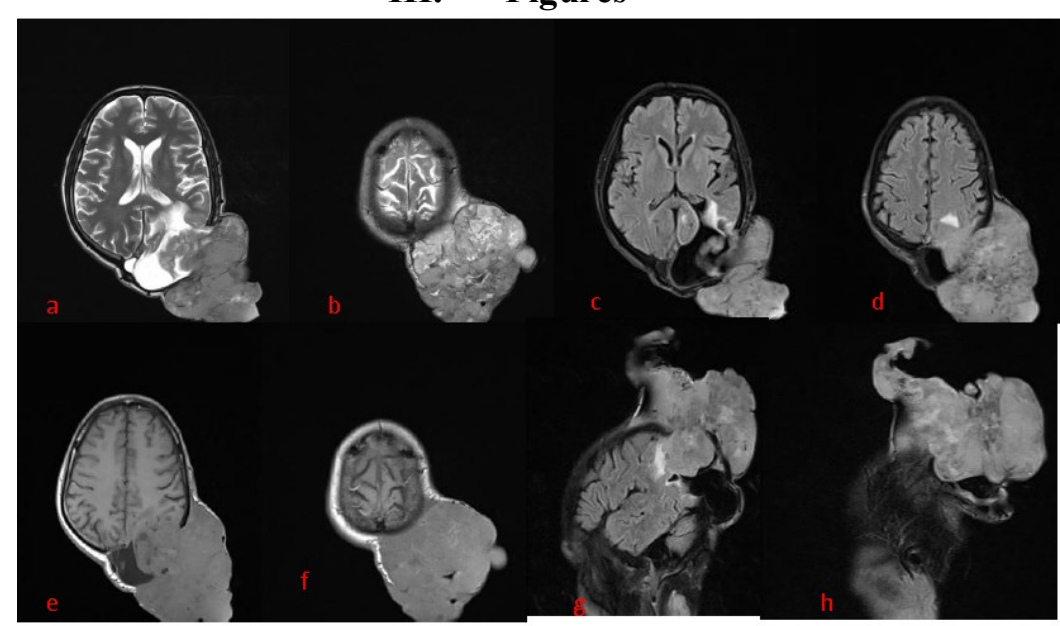

Fig1a,b) T2 weighted axial images show large predominantly isointense extra-axial mass lesion.c,d)FLAIR weighted axial images show similar findings to the T2images.

E,f) T1 weighted axial images shows isointense mass lesion, no evidence of haemorrhage within it.g,h) FLAIR sagittal images shows significant extracalvarial extension of the lesion.

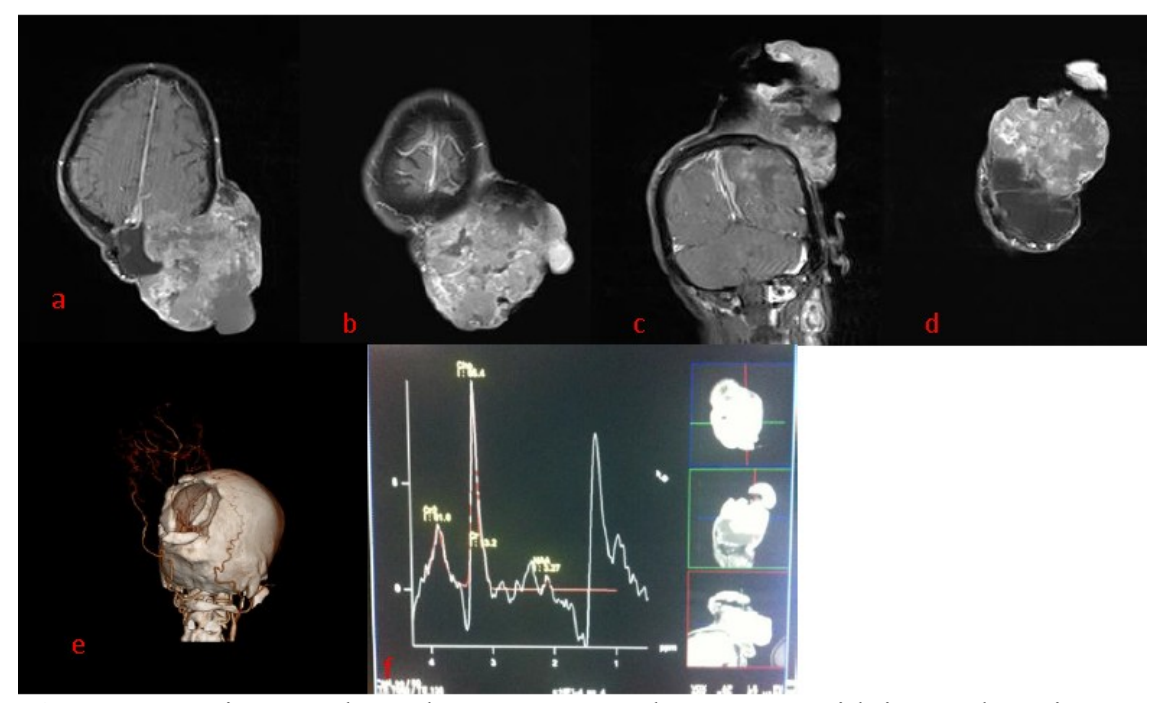

Fig 2 a,b,c,d) T1WI contrast images shows heterogenous enhancement with internal cystic areas.e)vrt images shows defect in the posterior skull with vessels supplying the lesion.

f)MRS shows significant choline, lipid and lactate peaks with near total dampening of NAA, Cr, MI peaks. 


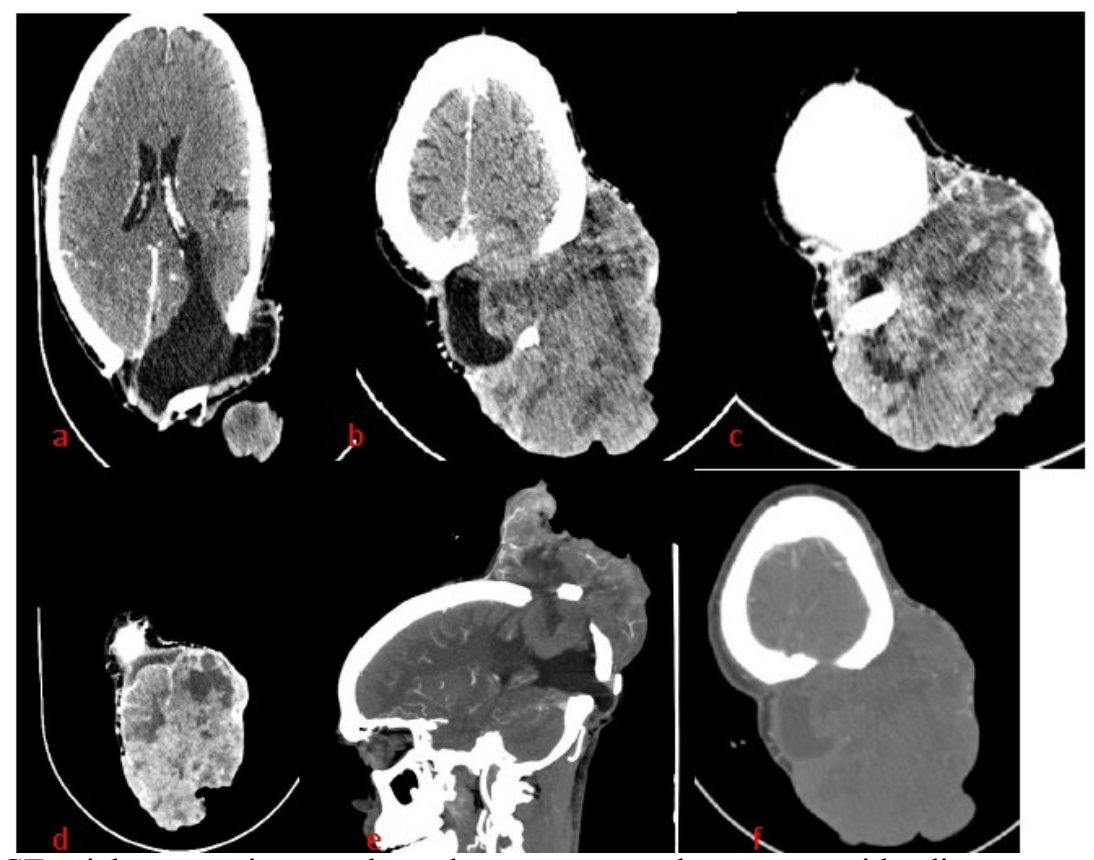

Fig 3.a,b,c,d) CT axial contrast images shows heterogenous enhancement with adjacent porencephalic cyst. e) CT contrast sagittal images shows lesion extending through the craniotomy site.

f) CT axial bone windows shows no evidence of calcification or bleed

\section{Discussion :}

Meningiomas arise from arachnoid meningothelial ("cap") cells. Between 50-60\% of meningiomas have inactivation of the NF2 gene product Merlin due to $22 q$ loss. Allelic losses on $1 p$ and $3 p$ are also common in sporadic meningiomas, particularly higher grade lesions.

Meningiomas can also be induced by radiation, with a dose-related time interval to tumor development that varies from 20 to 40 years. Many of these tumors have chromosome 7 monosomy.

Most meningiomas have progesterone or oestrogen receptors. Meningioma is one of the few brain tumors that exhibit a female predominance.

Meningiomas are graded into 3 grades by WHO.

I-Typical meningioma: these lesions are benign and account for $90 \%$ to $95 \%$ of lesions.

II-Atypical: these demonstrate intermediate hypercellularity and mitotic activity. They account for approximately $5 \%$ of lesions.

III-Anaplastic (malignant): they are characterized by necrosis, abundant mitoses, and parenchymal invasion. They account for $1 \%$ to $2 \%$ of meningiomas.

Histologically they can be divided into:-

- Syncytial

- Fibroblastic

- Transitional

- Psammomatous

- Papillary

- Malignant meningioma

- Sarcoma of the meninges

CT and MRI play important roles in the diagnosis of meningioma. Typically, meningiomas are sharply demarcated and hyperdense on CT. On MRI, the tumor is iso- or hypointense on non-contrast T1-W, and iso- or hyperintense on T2-W. Homogeneous enhancement is observed after contrast administration.

The imaging triad of extracranial mass, osteolysis, and "mushrooming" intracranial tumor is present in most but not all cases of Malignant meningiomas.

Malignant meningiomas also show adc values in the range of $(0.66 \pm 0.13 \times 10-3 \mathrm{~mm} 2 / \mathrm{s})$ which are significantly lower compared with benign meningiomas $(0.88 \pm 0.08 \times 10-3 \mathrm{~mm} 2 / \mathrm{s})$.

Intratumoral cysts and necrotic areas areas are also seen in malignant variant.

MRS shows significant choline peak, absent or reduced NAA or Cr peaks,increased alanine and glutamine peaks. 


\section{Conclusion:}

Anaplastic or malignant meningioma (MMen) corresponds histologically to WHO grade III.

Frankly malignant meningiomas are rare, representing only $1-3 \%$ of all meningiomas. MMens have a striking male predominance.

The imaging triad of extracranial mass, osteolysis, and "mushrooming" intracranial tumor is present in most but not all cases of Malignant meningiomas.

\section{Journal Papers:}

\section{References:}

[1]. Buetow M., Buetow P., Smirniotopoulos J. Typical, Atypical, and Misleading Features in Meningioma. RadioGraphics 1991; 11:1087-1 106.

[2]. Nagar VA, Ye JR, Ng WH, et al. Diffusion weighted MR imaging:diagnosing atypical or malignant meningiomas and detecting tumordedifferentiation. AJNR 2008; 29:1147-1152

[3]. Modha A, Gutin PH. Diagnosis and treatment of atypical and anaplastic meningiomas: a review. Neurosurgery 2005;57:538-50

[4]. Lee JH. Meningiomas, Diagnosis, Treatment, and Outcome. Springer Verlag. (2009) ISBN:1848829108

[5]. Grossman RI, Yousem DM. Neuroradiology, the requisites. Mosby Inc. (2003) ISBN:032300508X

[6]. Herrmann A et al: Proteomic data in meningiomas: postproteomic analysis can reveal novel pathophysiological pathways. J Neurooncol. 104(2):401-10, 2011

[7]. Vranic A et al: Mitotic count, brain invasion, and location are independent predictors of recurrence-free survival in primary atypical and malignant meningiomas: a study of 86 patients. Neurosurgery. 67(4):1124-32, 2010

[8]. Perry A et al: Meningiomas. In Louis DN et al: WHO Classification of Tumours of the Central Nervous System. 4th ed. Lyon, France: IARC Press. 164-72, 2007 